\title{
Joint Research on Scatterometry and AFM Wafer Metrology
}

\author{
Bernd Bodermann ${ }^{1}$, Egbert Buhr ${ }^{1}$, Hans-Ulrich Danzebrink ${ }^{1}$, Markus Bär ${ }^{1}$, Frank \\ Scholze ${ }^{1}$, Michael Krumrey ${ }^{1}$, Matthias Wurm ${ }^{1}$, Petr Klapetek ${ }^{2}$, Poul-Erik Hansen ${ }^{3}$, \\ Virpi Korpelainen ${ }^{4}$, Marijn van Veghel ${ }^{5}$, Andrew Yacoot ${ }^{6}$, Samuli Siitonen ${ }^{7}$, Omar \\ El Gawhary ${ }^{8}$, Sven Burger ${ }^{9}$, Toni Saastamoinen ${ }^{10}$ \\ ${ }^{1}$ Physikalisch-Technische Bundesanstalt (PTB), Braunschweig \& Berlin, Bundesallee 100, D-38116 Braunschweig \\ ${ }^{2}$ Cesky Metrologicky Institut Brno, Okružní 31, CZ-638 00 Brno \\ ${ }^{3}$ Dansk Fundamental Metrolog, Matematiktorvet 307, DK-2800 Kongens Lyngby \\ ${ }^{4}$ Mittatekniikan Keskus, Tekniikantie 1, P.O. Box 9, FI-02151 Espoo \\ ${ }^{5}$ VSL B.V., Thijsseweg 11, P.O. Box 654, NL-2629 JA Delft \\ ${ }^{6}$ National Physical Laboratory, Hampton Road, Teddington, Middlesex, TW11 OLW \\ ${ }^{7}$ Nanocomp Oy Ltd, Ensolantie 6, FI-80710 Lehmo \\ ${ }^{8}$ Delft University of Technology, Lorentzweg 1, NL-2628 CJ Delft \\ ${ }^{9} J C M$ wave GmbH, Bolivarallee 22, D-14050 Berlin \\ ${ }^{10}$ University of Eastern Finland, Yliopistokatu 7, FI-80100 Joensuu
}

\begin{abstract}
Supported by the European Commission and EURAMET, a consortium of 10 participants from national metrology institutes, universities and companies has started a joint research project with the aim of overcoming current challenges in optical scatterometry for traceable linewidth metrology. Both experimental and modelling methods will be enhanced and different methods will be compared with each other and with specially adapted atomic force microscopy (AFM) and scanning electron microscopy (SEM) measurement systems in measurement comparisons. Additionally novel methods for sophisticated data analysis will be developed and investigated to reach significant reductions of the measurement uncertainties in critical dimension (CD) metrology. One final goal will be the realisation of a wafer based reference standard material for calibration of scatterometers.
\end{abstract}

Keywords: Scatterometry, CD metrology, AFM, reference standard, rigorous modelling, inverse diffraction problem PACS: 06.30.Bp, 06.20.fb, 07.05.Tp, 07.79.Lh, 81.16.Nd

\section{INTRODUCTION}

The development of sophisticated dimensional metrology is essential to meet the metrology requirements for future technology needs. CDmetrology in particular is identified by the ITRS ${ }^{1}$ as an essential challenge. For production measurements scatterometers and CD-SEMs are key metrology tools. Although measurement results of these different tools usually show a good correlation, systematic offsets of the order of few $10 \mathrm{~nm}$ are often observed (fig. 1). Universal product-related reference standards for characterisation and calibration of scatterometers are currently not available.
Within the European Metrology Research Program [1] (EMRP) a joint research project (JRP) with 10 participants from European national metrology institutes, universities and companies is jointly supported by the European Commission and the participating countries within the European Association of National Metrology Institutes (EURAMET e.V.) [2]. The aim of this project is to overcome current challenges in optical scatterometry. This JRP will significantly improve and extend scatterometry methodically and regarding application areas. These extensions include the application of short wavelengths down to X-rays, a stringent exploitation of the polarisation degree of freedom, using phase information of the scattered field by scanning with a focused coherent spot and new

\footnotetext{
${ }^{1}$ International Technology Roadmap for Semiconductors

Frontiers of Characterization and Metrology for Nanoelectronics: 2011 AIP Conf. Proc. 1395, 319-323 (2011); doi: 10.1063/1.3657910 (C) 2011 American Institute of Physics 978-0-7354-0965-1/\$30.00
} 
detection schemes together with the development of improved or new concepts for modelling and data analysis.

a)

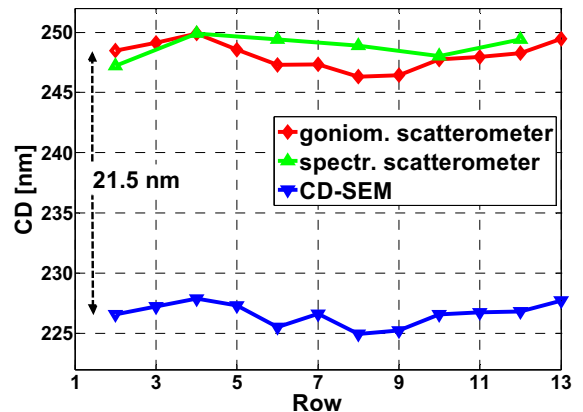

b)

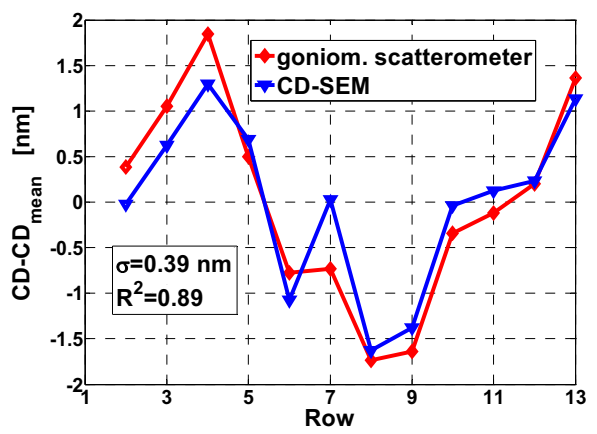

FIGURE 1. a) Typical measurement example of nominally equal CDs on different locations (row 2 13) on a state of the art high-end COG photomask using a commercial scatterometer (green), a commercial CD-SEM (blue) and PTBs goniometric DUV scatterometer [3]; b) the difference between measured and mean $\mathrm{CD}$ indicates the good $\mathrm{CD}$ linearity between the results obtained with PTBs scatterometer and the commercial CD-SEM

For further refinement of the scatterometric algorithms, the impact of the structure form and local deviations must be taken into account. The necessary input on structure details will be provided by high resolution SEM and AFM methods, which have to be expanded to provide comparability to scatterometric measurements.

Finally a scatterometry reference standard will be designed, developed, characterised and calibrated to face the tough specifications demanded by the semiconductor industry for future technology needs. As a first step towards a "golden reference standard" suitable for calibration of scatterometers this standard will be designed and developed to be suitable also for testing of AFMs and SEMs, so that the matching of these different tools will be possible.

Thus not only a comparability of these methods is introduced but also a mutual support of the partly redundant and partly supplementing information obtained by the different methods is provided. Sophisticated data analysis schemes will be developed in this JRP to reach levels of measurement uncertainty for different measurands significantly beyond the uncertainty levels reachable for each individual metrology method.

Additionally this project combines and extends the current scatterometry application fields towards surface roughness, periodically structured surfaces and diffractive optics, so that the synergy between metrology for semiconductor industry and for optics industry will be exploited.

\section{DEVELOPMENT OF ADVANCED SCATTEROMETRY}

An essential part of the research within this project aims to overcome the current limitations of applications of scatterometry. One main goal is to establish scatterometry as traceable and absolute metrological method for dimensional measurements of micro- and nanostructures on surfaces $[4,5]$. Recent investigations [6] have shown, that even small feature details like line edge roughness (LER) or line widths roughness (LWR) do significantly affect the analysis of scatterometric measurements. Therefore, the scatterometric fields of application shall be extended from basic measurands like $\mathrm{CD}$, pitch or side wall angle to a significantly more detailed structure description including e.g. corner rounding, footing, roughness (both surface, LER and LWR) and further edge profile details, if appropriate. To provide the sensitivity required especially in the high spatial frequency regime, both the exploitation of the advantages of using shorter wavelengths ranging from the deep over extreme ultraviolet (DUV, EUV) down to X-rays [7, 8], and the development and exploration of new methods like Fourier scatterometry, Mueller polarimetry will be investigated. Further on, scatterometry shall be extended to reliable metrology of 3D structures like e.g. contact hole arrays in lithography or diffractive optics. Finally, well known limitations and challenges like the limited interaction area, influence of local structure parameter variations on the integral scatterometric measurements, the possible interference between structuring and other production parameters and the complex refractive index of the structure materials will be studied thoroughly and a concept to overcome or to take into account these effects will be developed and tested.

Comparison measurements will be performed applying both these novel scatterometry methods and conventional scatterometry methods on different test samples both with 1D and 2D grating structures to compare the metrological potential of each method, to test their respective sensitivity regarding the different structure details and to evaluate the results with respect to traceability of scatterometry. 


\section{HIGH RESOLUTION AFM AND SEM METHODS}

AFM and SEM metrology systems will be applied as reference tools for the characterisation of structural details such as roughness, edge profiles or corner roundings. In addition they will support the validation of absolute and traceable scatterometry, e.g. high resolution measurement of the widths of nanostructures. Scatterometers perform integral measurements over a relatively large interaction area of typically $(100 \mu \mathrm{m})^{2}$. However, no local information about individual structures is obtained. This information will be acquired by AFM and SEM, which are limited due to longer measurement times and smaller measurement fields.

To enable a comparison between the SEM/AFM and scatterometric measurements special sampling schemes (e.g. measuring long rectangular scanning fields in different orientations) and data analysis methods have to be developed and applied.

With a well-characterised probe and a translation stage using state-of-the-art interferometric displacement measurement, AFMs can be linked to the SI traceability chain. In this way they can provide information on the geometry of samples which is traceable to the SI unit of length with a well-defined uncertainty. This makes AFM invaluable as a reference tool to support the other techniques. The main challenges which will be addressed are measuring 1D pitch over long ranges with high accuracy and resolution and measuring local variations in the 3D shape of nano-scale structures. A facility for ultra-high accuracy 1D pitch measurements using a combination of X-ray and optical interferometry (fig. 2) [9] together with an AFM will meet the first challenge.

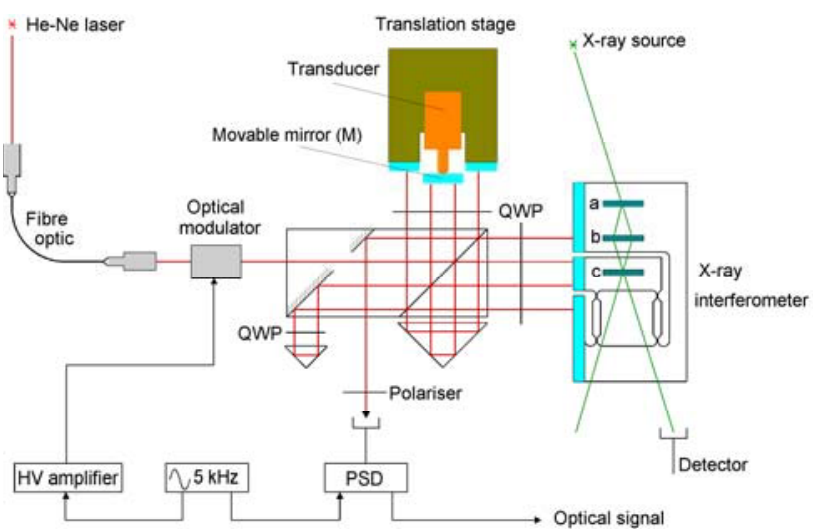

FIGURE 2. Scheme of a combined optical \& $x$ ray interferometer (COXI), which will be combined with an AFM head
To meet the second challenge, a special long-range 3D-AFM will be developed and used to measure local shape variations. These measurements provide local detail information of individual structures which are important input data for the numerical modelling of scatterometry, for the validation of scatterometric results, for testing the limits of scatterometry and for the characterisation of standard artefacts described below.

\section{IMPROVED DATA EVALUATION AND NUMERICAL MODELLING OF THE INVERSE DIFFRACTION PROBLEM}

The accuracy of scatterometric measurements is crucially dependent on advanced modelling and sophisticated data analysis. For the solution of the inverse problem [10] at the heart of scatterometry, the quality of the employed model, its unavoidable systematic effects on the overall measurement uncertainty, and the correct evaluation of input data represent important factors for the determination of nanoscale surface profiles together with their associated measurement uncertainties [11]. Altogether, improvement of modelling and data analysis shall establish a better mapping between the measured optical properties and the searched geometric parameters of the surface structure.

Two central goals are the implementation of statistical methods for advanced uncertainty analysis and the improvement of simulation algorithms in order to overcome present limitations especially in numerical speed, accuracy and model complexity.

This will be done on the basis of numerical modelling of electromagnetic field scattering and the near and far field distribution. Different numerical methods, in particular the Finite Element Method, the Finite Difference Time Domain Method, and the Rigorous Coupled Wave Analysis, will be employed and compared with each other regarding their speed, efficiency and real-time capability.

An important part of the effort will be devoted to developing more realistic models, related numerical algorithms and to carrying out the corresponding simulations that will describe the experimental measurements even for non-ideal cases (non-periodic spatial components, input beam geometry and polarisation state, various material properties). Besides the named standard numerical methods new and improved rigorous methods like offline-online decomposition schemes for parameterised setups [12] will be developed and applied to increase the capabilities to handle more complex structure models. This shall lead to a better correspondence of 
simulations with real samples measurements and will enable a proper analysis of all imperfections, irregularities and existing model approximations. Additionally, increased capability in terms of numerical speed and accuracy will facilitate to avoid significant influences of numerical errors on the error budget.

The improved modelling and data analysis will be applied to the scatterometry measurements mentioned above.

\section{DEVELOPMENT OF A SCATTEROMETRY STANDARD}

An essential goal within this project is to develop reference standards for metrology in semiconductor industry, in particular for wafer processing including lithography. The final goal is the equalisation and matching of the various metrology technologies (scatterometry, SEM and AFM) as used for process development and process control in the lithographic industry for the two important measurands $\mathrm{CD}$ and overlay. To reach this goal it is necessary to realise as a long-range objective, a so-called 'golden' reference standard, which should be useable equally for AFM, SEM and scatterometry. Although there exist standards applicable for AFM and SEM, these standards are not suitable for scatterometry, mainly because the overall size of the structured area is too small. Moreover, there is not even a suitable standard available for applications in high-end scatterometry.

Therefore within this project first and important steps towards the direction of such a golden reference standard shall be achieved. In a first approach, two specialised scatterometry standards will be designed, characterised and calibrated. Various metrological requirements in semiconductor industry shall be gathered and aligned to design standards that cover important applications. Thus different type of standard samples and materials will be necessary to cover different metrological applications. For example for resist metrology a material is required, which mimics the optical properties of common resist materials, while for inspection of the fabricated wafers a $\mathrm{Si}$ standard would be most meaningful. The requirements and specifications of the standards will be discussed and aligned with partners from industry and stakeholders. Two types, a Si- and a resist mimicking standard, will be developed. The standards will be designed such that they are measurable also by microscopic methods (SEM, AFM). In the long-run (but outside the scope of the project) the standards shall be extendable to a golden reference standard.

The scatterometry standards have to meet a number of requirements and boundary conditions:
- The design (sample size, materials and structuring) needs to take into account the different requirements of scatterometers, SEMs and AFMs

- The structures shall provide a set of targets representative for current lithography technologies

- The standards should have the potential to be extendable to future technology steps $(22 \mathrm{~nm}$ node and beyond). That means, that, if possible, the materials should be selected such that they can be processed and used in future technology.

- An accurate knowledge of the optical parameters of the materials is necessary for scatterometric characterisations; applicability for SEM and AFM requires a good knowledge of electrical and mechanical properties.

- For industrial applications, the standards have to be suitable for state of the art metrology tools. Today, these systems usually handle $300 \mathrm{~mm}$ wafers and the applied scatterometers require a minimum size of the scatterometry target of about $0.1 \times 0.1 \mathrm{~mm}^{2}$. However, the calibration tools used at the NMIs in most cases cannot handle $300 \mathrm{~mm}$ wafers and, compared to standard samples for the high resolution microscopy tools, the scatterometer targets are huge.

- The standards shall be stable over time.

The development of a suitable standard requires an iterative process of succeeding optimisation steps. Depending on the industrial requirements, tests of different materials could be necessary. A well-controlled manufacturing is of key-importance in view of high-reproducibility.

The samples will be characterised thoroughly: structure details like roughness, edge profiles and edge angles will be characterised systematically by AFM, SEM and Grazing Incidence Small Angle Scattering (GISAXS) measurements. Finally, the samples will be calibrated to become a reference standard. For this purpose a combination of different scatterometry, AFM, SEM as well as GISAXS and EUV scatterometry measurements will be used. Each of these methods shall provide those parameters which were found to be most reliably determined by each of them. The measurement uncertainty required by semiconductor industry in CD-metrology is about $1 \%$ of the node-value (e.g. $0.3 \mathrm{~nm}$ for $32 \mathrm{~nm}$ node). Such tough requirements can only be met by the combination of the different measurement methods developed here and by applying the sophisticated combined data analysis methods. The data analysis will be supported by information about different structure details as provided by the AFM measurements. 


\section{APPLICATION OF SCATTEROMETRY ON DIFFRACTIVE AND DIFFRACTIVE REFRACTIVE OPTICAL ELEMENTS}

In recent investigations scatterometry has been identified as a well-suited metrology tool for characterizing challenging structured surfaces such as high aspect gratings, slanted gratings and gratings on curved surfaces [13]. Going from planar to curved structure changes the optical response from a pure diffractive response to a combined diffractive and refractive response. This transition changes key parameters in planar scatterometry like a uniform pitch. Introducing curvature leads to a non-uniform pitch that depends on the local curvature, making it impossible to use the usual simplified scatterometry approach of a repeated unit cells. Curvature also changes the diffraction properties of the structure, which in this case has contributions from both the reflective curvature and the nanostructures placed on top of it. The reflective curvature can be many times larger than the size of the nanostructures placed on top of it, making it a huge numerical challenge to perform direct calculation of the diffraction properties of the curved structure. The complexity of diffractiverefractive optical components compared to planar structure makes it advantageous to measure more Mueller matrix elements than usually done in conventional scatterometry. Therefore Mueller Polarimetry, which is capable of measuring the full Mueller matrix of the structure, will be used. The access to significantly more independent measurement information than conventional scatterometry will be used to obtain the dimensional parameters by using a $\chi^{2}$ best-fit matching procedure between calculated and measured lineshape curves $[14,15]$. It is expected that Mueller Polarimetry will significantly improve the estimation of the dimensional parameters compared with conventional scatterometry. Suitable test samples with flat and curved surfaces will be investigated using optical scatterometry and Mueller polarimetry to explore the limits of measuring dimensional and optical properties of 2D and 3D nanostructures using scatterometric-optical techniques.

\section{ACKNOWLEDGMENTS}

This project is supported by a manifold of collaborators from industry, universities and research institutes. We would like to thank all collaborators, in particular ASML, Carl Zeiss AG and NIST for their support, their scientific-technical input and for fruitful discussions in the forefront of this project and for the willingness to sustain this support during the project runtime. We thank the European commission and the EURAMET e. v. for financial support under the support code No 912/2009/EC.

\section{REFERENCES}

1. http://www.emrponline.eu

2. http://www.euramet.org

3. M. Wurm, F. Pilarski, B. Bodermann: A new flexible scatterometer for critical dimension metrology. Review of Scientific Instruments 81, 023701 (2010)

4. Frank Scholze, Bernd Bodermann, Hermann Groß, Akiko Kato, Matthias Wurm: First steps towards traceability in scatterometry, Proc. SPIE 7985, (2011), $79850 \mathrm{G}$

5. Heather J. Patrick, Thomas A. Germer, Progress toward traceable nanoscale optical critical dimension metrology for semiconductors, Proc. SPIE 6672, (2007), 66720L

6. Akiko Kato, Frank Scholze: Effect of line roughness on the diffraction intensities in angular resolved scatterometry, Appl. Opt. 49 (2010), 6102-6110

7. F. Scholze and C. Laubis: Use of EUV scatterometry for the characterization of line profiles and line roughness on photomasks. EMLC 2008, 374-382, VDE VERLAG GMBH Berlin und Offenbach (2008)

8. M. Krumrey, G. Gleber, F. Scholze and J. Wernecke: Synchrotron radiation-based X-ray reflection and scattering techniques for dimensional nanometrology Meas. Sci. Technol. (accepted for publication)

9. A. Yacoot, L. Koenders: From Nanometre to Millimetre: A Feasibility Study of the Combination of Scanning Probe Microscopy and Combined Optical and X-ray Interferometry. Meas. Sci. Technol. 14, (2003) N59-63

10. H. Groß, R. Model, M. Bär, M. Wurm, B. Bodermann and A. Rathsfeld, Mathematical modelling of indirect measurements in periodic diffractive optics and scatterometry. Measurement 39, (2006) 782-794.

11. H. Groß, A. Rathsfeld, F. Scholze and M. Bär, Profile reconstruction in extreme ultraviolet (EUV) scatterometry: modeling and uncertainty estimates. Meas. Sci. Technol. 20, (2009) 105102 - 105112.

12. J. Pomplun, F. Schmidt: Accelerated A Posteriori Error Estimation for the Reduced Basis Method with Application to $3 D$ Electromagnetic Scattering Problems SIAM J. Sci. Comput. 32 (2010) 498 - 520

13. J. Garnaes P.-E. Hansen, N. Agersnap, J. Holm, F. Borsetto, and A. Kühle: Profiles of high aspect ratio grating determined by optical diffraction microscopy and atomic force microscopy. Appl. Opt. 45, (2006), 32013212

14. P.-E- Hansen, L. Nielsen: Combined opterimization and hybrid scalar-vector diffraction method for grating topography parameters determination. Mat. Sci. and Eng. B 165, (2009), 165-168.

15. M. Karamehmedovic, M. P. Sørensen , P.-E- Hansen, A. V. Lavrinenko: L. Nielsen: Application of the method of auxiliary sources to a defect-detection inverse problem of optical diffraction microscopy. J. Eur. Opt. Soc. Rapid Publ. 5, (2010), 10021-1 to 10021-7 